\title{
IMPROVEMENTS IN PATIENT TREATMENT PLANNING SYSTEMS
}

\author{
Floyd J. Wheeler ${ }^{\mathrm{a}}$, Daniel E. Wessol ${ }^{\mathrm{a}}$, Ray Babcock ${ }^{\mathrm{b}}$, David W. Nigg ${ }^{\mathrm{a}}$, Carol A. Atkinson ${ }^{\mathrm{a}}$, and \\ John Evans \\ a'daho National Engineering Laboratory, Idaho Falls, ID, 83415-3890 USA, 208-526-7641. \\ bMontana State University, 410 Roberts Hall/CS Department, Bozeman, MT, 59717 USA, \\ 406-994-3707.
}

\section{INTRODUCTION}

The Boron Neutron Capture Therapy, Radiation treatment planning environment (BNCT_Rtpe) software system is used to develop treatment planning information ${ }^{2}$. In typical use BNCT_Rtpe consists of three main components: (1) Semi-automated geometric modeling of objects (brain, target, eyes, sinus) derived from MRI, CT, and other medical imaging modalities, (2) Dose computations for these geometric models with rtt_MC, the INEL Monte Carlo radiation transport computer code, and (3) Dose contouring overlaid on medical images as well as generation of other dose displays. We continue to develop a planning system based on tbree-dimensional image-based reconstructions using Bspline surfaces. Even though this software is in an experimental state, it has been applied for large animal research and for an isolated case of treatment for a human glioma. Radiation transport is based on Monte Carlo, however there will be implementations of faster methods (e.g. diffusion theory) in the future. The important thing for treatment planning is the output which must convey, to the radiologist, the deposition of dose to healthy and target tissue. Many edits are available such that one can obtain contours registered to medical image, dose/volume histograms and most information required for treatment planning and response assessment. Recent work has been to make the process more automatic and easier to use. The interface, now implemented for contouring and reconstruction, utilizes the Xwindowing system and the MOTIF graphical users interface for effective interaction with the planner. Much work still remains before the tool can be applied in a routine clinical setting.

\section{APPLICATION OF THE SOFTWARE}

Initial canine epithermal-neutron irradiation research was based on predictions of dose rates at the location of peak thermal flux in tissue. These peak values and dose/depth data can be calculated quite accurately given detailed knowledge of the beam characteristics and patient geometry. When biological response of dogs irradiated in different field geometries was compared, however, it became apparent that peak dose is not adequate for prediction of response. Dose contours, presented on medical image, are necessary as well as dose/volume histograms and three-dimensional displays. For example, Figures 1 and 2 show dose contours and dose/volume histogram for beagles irradiated in the High Flux Reactor epithermal beam at Petten, The Netherlands ${ }^{2}$, and Figures 3 and 4 show the same for Labrador dogs irradiated in the Medical Research Reactor (BMRR) epithermal beam at Brookhaven, USA ${ }^{3}$. Planning for these irradiations was based upon peak dose for both cases but positive healthy tissue response was found at lower (by 30\%) equivalent peak dose for the Petten beagles. Examination of the isodose contours over image and the dose/volume histograms helps to explain the difference. For the Petten beagles, an 8-cm circular beam was centered over the beagle brain (which has significantly less cover tissue than the Labrador brain). For the Labrador dogs, irradiation was accomplished with a $5-\mathrm{cm}$ by 10-cm rectangular beam centered over the right hemisphere. The isocontours show the peak in the beagle brain to be close to brain center whereas the peak is actually outside the brain in the case of the Labrador. Also, comparison of the dose/volume histograms show a large difference in volume of brain irradiated at higher dose levels. These dogs were administered BSH and the calculated

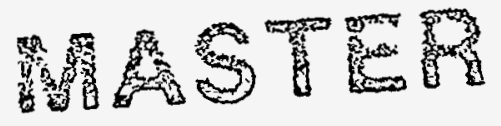




\section{DISCLAIMER}

Portions of this document may be illegible in electronic image products. Images are produced from the best available original document. 
doses are given for a blood concentration of $50 \mathrm{ppm}$.

Figure 1. Total physical dose isocontours for the Petten beagle with $50 \mathrm{ppm}{ }^{10} \mathrm{~B}$.

Figure 2. Dose/volume histogram for the Petten beagle for the brain with $50 \mathrm{ppm}{ }^{10} \mathrm{~B}$.

Figure 3. Total physical dose isocontours for the BMRR labrador with $50 \mathrm{ppm}{ }^{10} \mathrm{~B}$.

Figure 4. Dose/volume histogram for the BMRR labrador for the right hemisphere with $50 \mathrm{ppm}$ ${ }^{10} \mathrm{~B}$.

A conservative irradiation for the human epithermal irradiation (using BPA-F) was performed using a single-field $8-\mathrm{cm}$ beam directed at the target. Figure 5 shows dose contour over a region map of the model for equivalent dose to tumor cells and Figure 6 shows dose/volume information for healthy brain. Peak equivalent dose to healthy tissue was predicted to be $10 \mathrm{~Gy}$. It will be some time before interpretation of response can be made but it is obvious that peak dose will not be adequate for a full understanding of the response of both tumor and healthy tissue.

Figure 5. Tumor equivalent dose isocontours for BMRR glioma patient with assumed ${ }^{10} \mathrm{~B}$ tumor concentration of $30 \mathrm{ppm}$. BPA-F Compound Factor set to 3.8, proton RBE set to 3.2.

Figure 6. Dose/volume for healthy brain for a BMRR glioma patient with ${ }^{10} \mathrm{~B}$ blood concentration of $11.2 \mathrm{ppm}$. Here, the healthy brain BPA-F Compound Factor is assumed to be 1.3, and the proton (fast and $14 \mathrm{~N}$ ) RBE is set to 3.2 .

\section{DISCUSSION}

A complete assessment of tumor and healthy-tissue response will require availability of detailed calculation results displayed in formats interpretable by clinicians and researchers. For clinical applications, software must be available to rapidly generate point, line, and volume information for any region of interest for an image-based model of the patient. Development of the Rtpe system remains directed toward that goal. Rtpe now has capability to provide information required for treatment planning and development is proceeding to reduce planner and computer time requirements. Presently, about one week lead time is required for a treatment plan and a complete calculation for one field requires about 2 computer hours on an HP 700/125 workstation. With experience, a plan could probably be developed in three days with the present system, and with development, this could be reduced to about one day in perhaps one year from now. Recent work has been in validation and additions of features that automate the process and reduce the occurrence of error due to user actions. For example, it is very easy to generate isocontours over image or over model. The user simply specifies the file name of an image, the field of view, a scale factor and the perpendicular spatial coordinate and the rtt_MC module generates the corresponding contour data and a region overlay mask. The mask is useful to check the registration of data to image and model to image and for clipping off contours lying outside the area of interest. Also, a start at automated plots has been made. For line plots and dose/volume plots, the user can obtain a graphical display by specifying a one-line command. The system responds by popping up a window for each plot where the current data is presented on a standard plot template. Software has also been written to allow multi-field irradiations. The present software for multi-field is just a crude accounting package to weight various fields by power/time and boron concentration. In the future, a planning tool will present a user-friendly window to allow the planner to rapidly select fields and fractions and view results in interactive mode. 


\section{REFERENCES}

1. D.E. Wessol, et al. "Users Manual for the BNCT_Rtpe system", to be published.

2. K.H.I Philipp, R. Huiskamp, J. Casado, and R. L. Moss, "Normal Tissue Tolerance in a Large Animal Model of Boron Neutron Capture Irradiation with Borocaptate Sodium (BSH)", these proceedings.

3. P. R. Gavin, S. L. Kraft, C. E. DeHaan, C. D. Swartz, and M. L. Griebenow, "Large Animal Normal Tissue Tolerance with Boron Neutron Capture," International Journal of Radiation

Oncology Biology Physics, 28, No. 5, 1099-1106, (1994).

\section{DISCLAIMER}

This report was prepared as an account of work sponsored by an agency of the United States Government. Neither the United States Government nor any agency thereof, nor any of their employees, makes any warranty, express or implied, or assumes any legal liability or responsibility for the accuracy, completeness, or usefulness of any information, apparatus, product, or process disclosed, or represents that its use would not infringe privately owned rights. Reference herein to any specific commercial product, process, or service by trade name, trademark, manufacturer, or otherwise does not necessarily constitute or imply its endorsement, recommendation, or favoring by the United States Government or any agency thereof. The views and opinions of authors expressed herein do not necessarily state or reflect those of the United States Government or any agency thereof. 


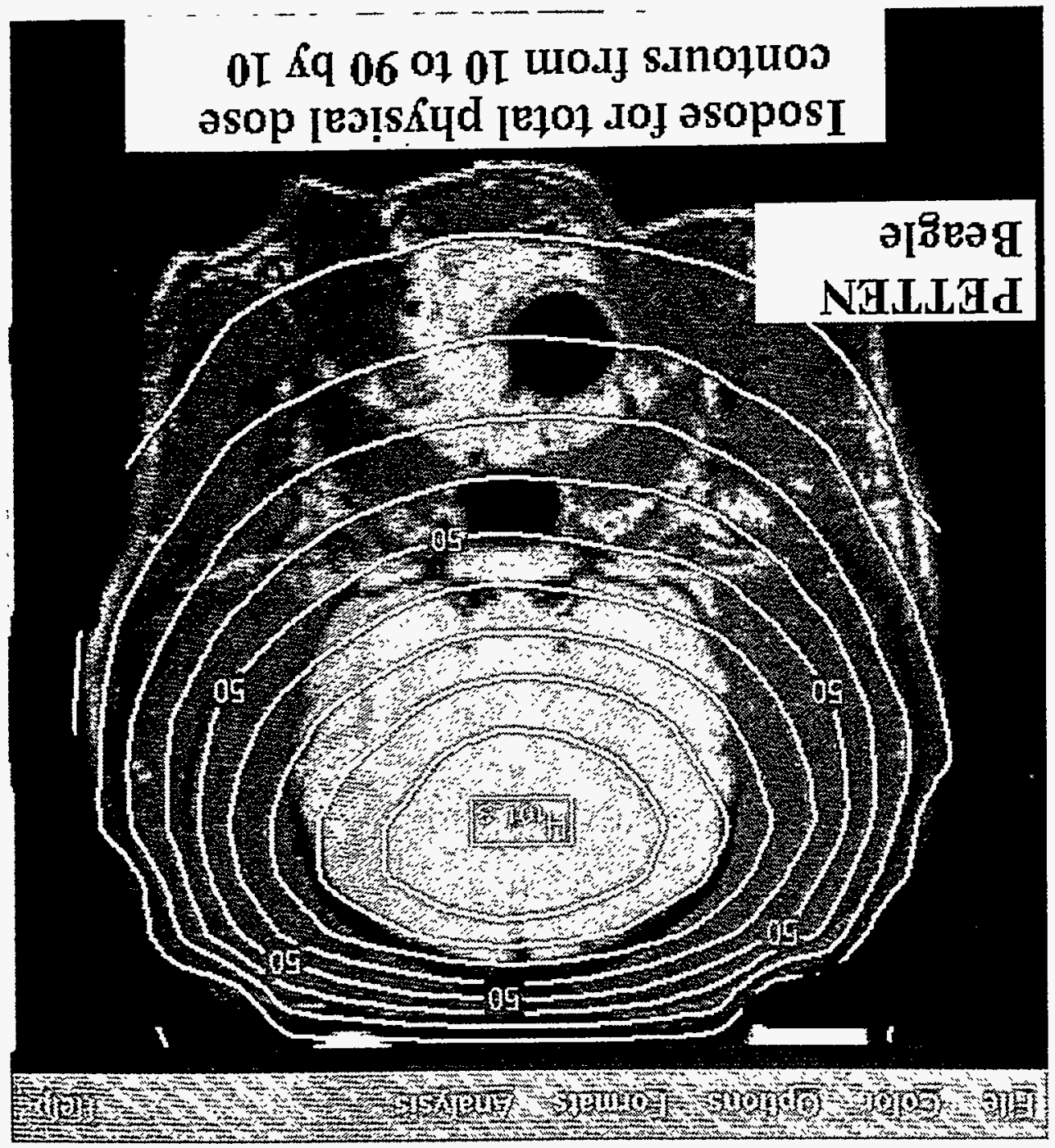




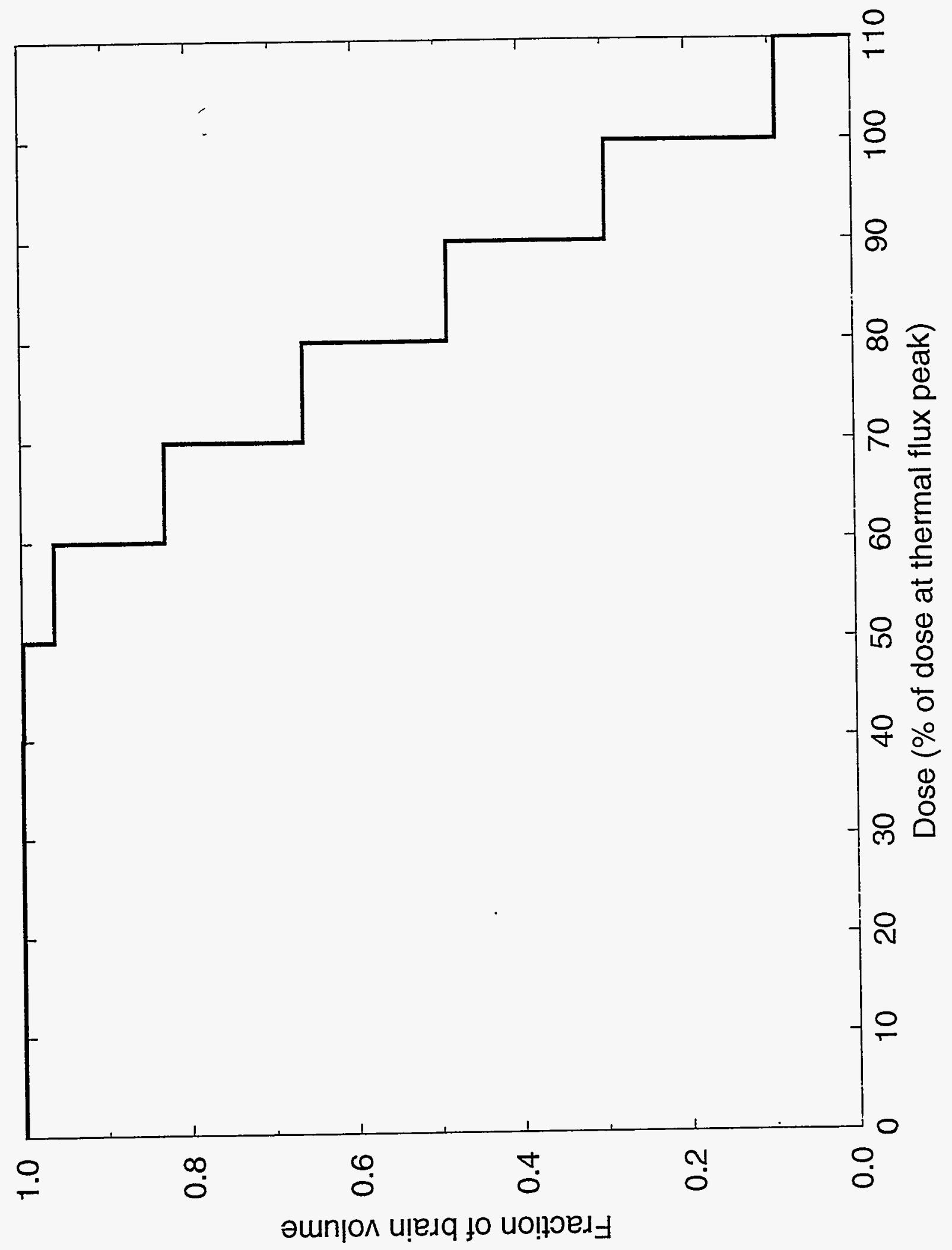




\section{(org urdd 0S) OI $\mathrm{Aq}$ O0I of OI UIO.J sInoquos asop [eoIskud [eqǫ do] asoposI}

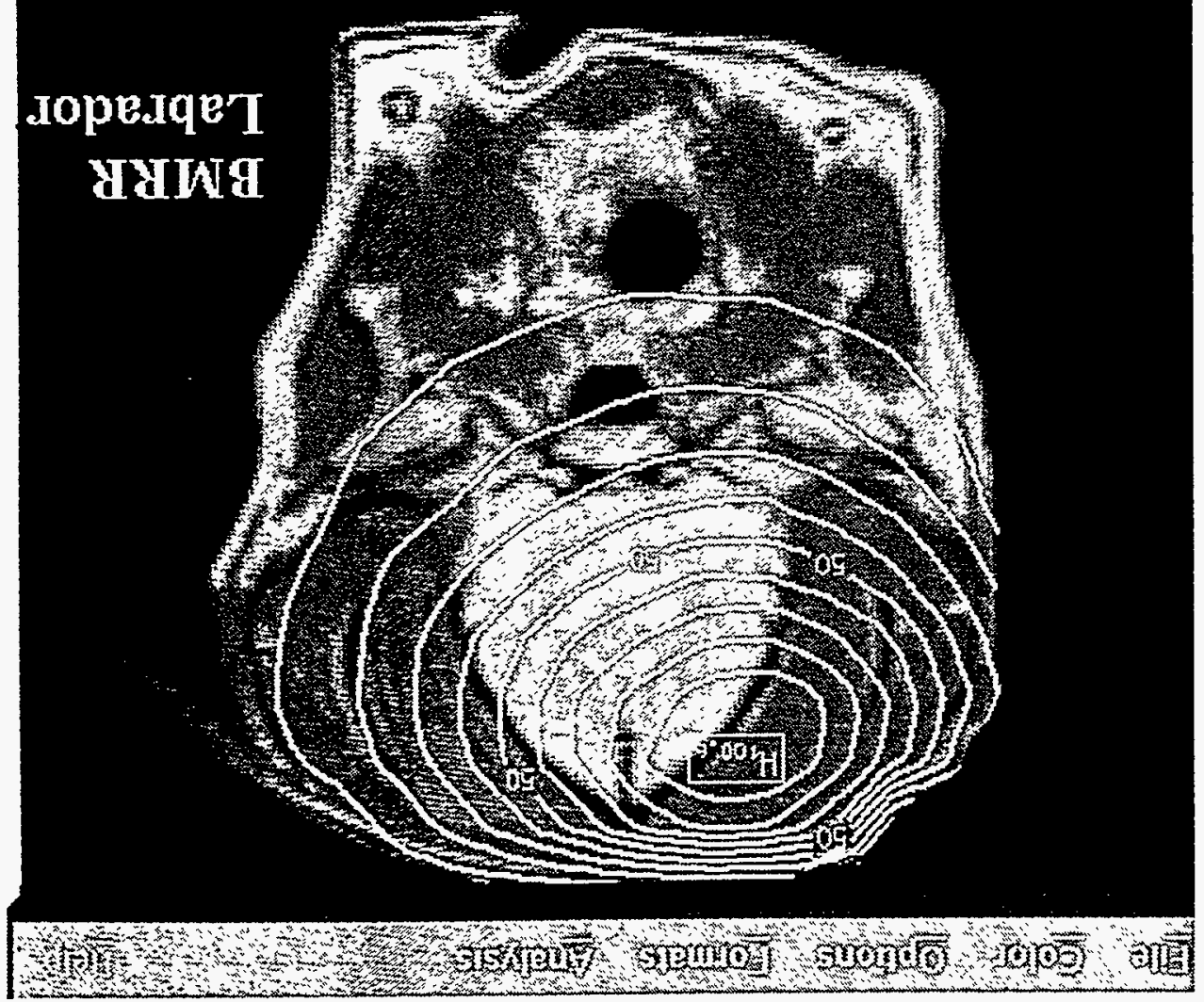

4 


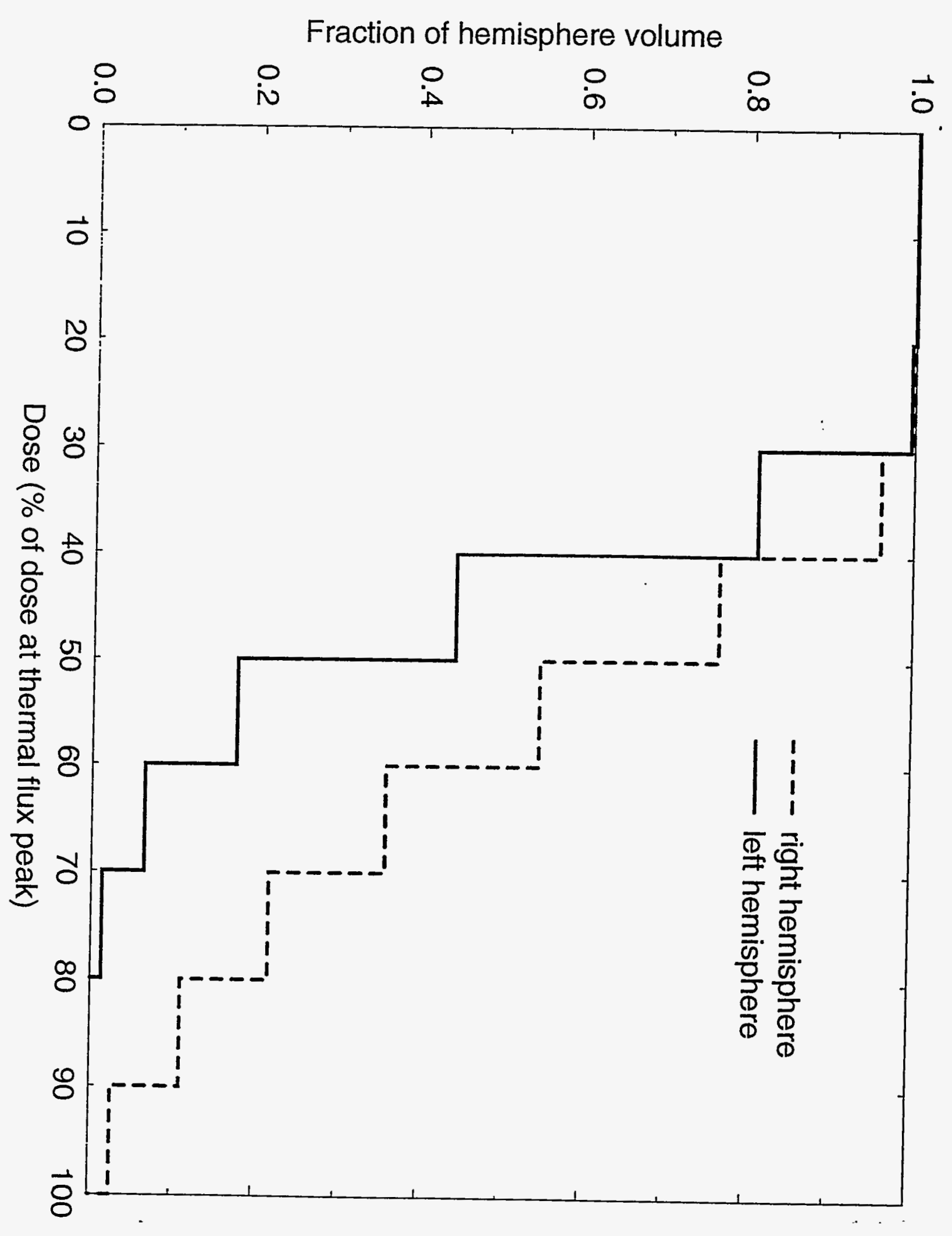




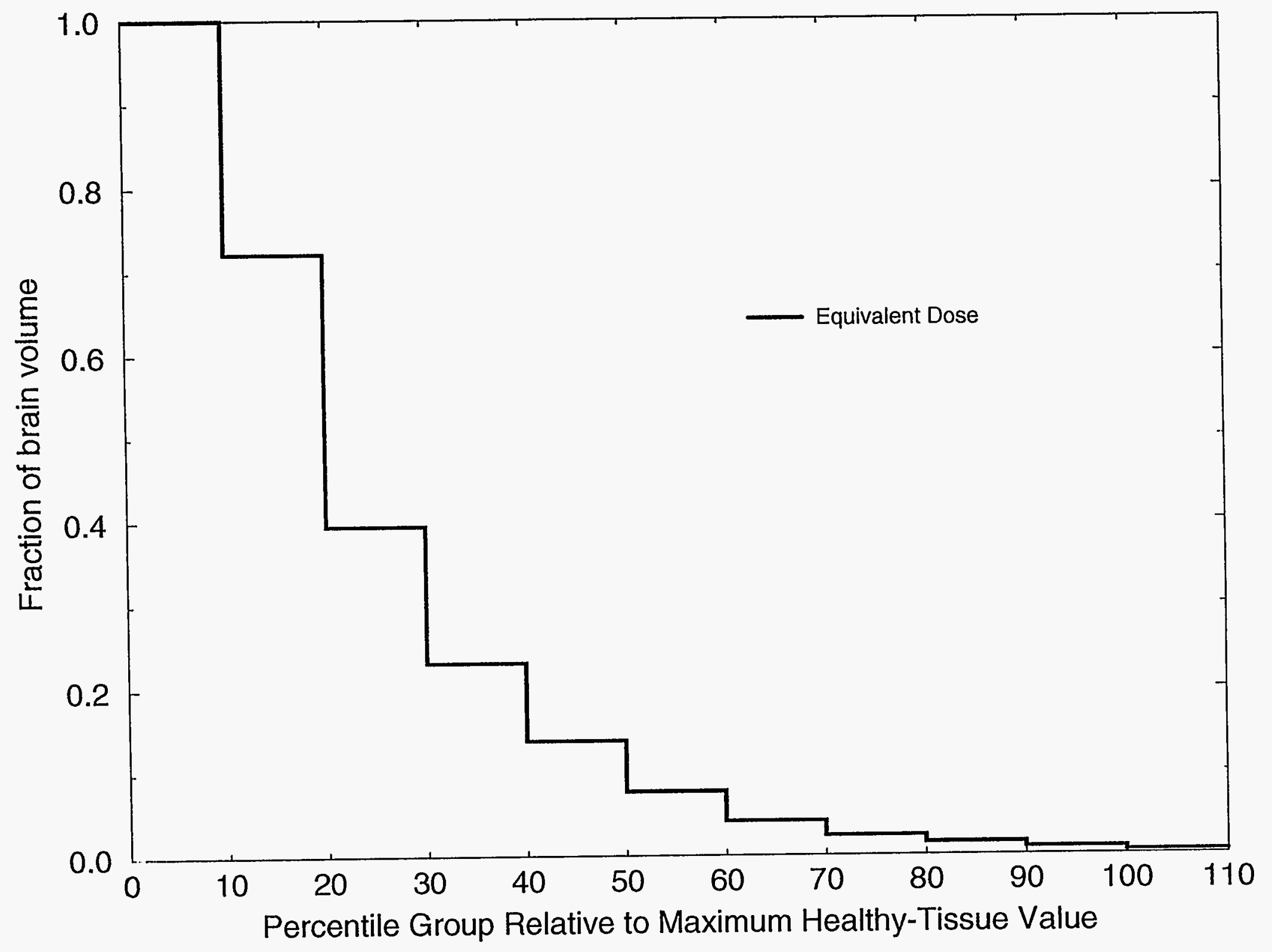

